\title{
Checklist of seaweeds of Al-Hoceima National Park of Morocco (Mediterranean Marine Protected Area)
}

\author{
Hanaa Moussa', 2, Mustapha Hassoun', Ghizlane Salhi', Hanaa Zbakh1 \& Hassane Riadi ${ }^{1}$ \\ ${ }^{1}$ Applied Phycology-Mycology Group, Applied Botany Laboratory, Department of Biology, Faculty of Sciences, Abdelmalek \\ Essaâdi University, M'Hannech II, 93030 Tétouan, Morocco \\ ${ }^{2}$ Department of Pharmacology, Faculty of Pharmacy, University of Santiago de Compostela, 15782 Santiago de \\ Compostela, Spain
}

\section{Correspondencia}

H. Moussa

e-mail: moussa.hanaa-etu@uae.ac.ma

Recibido: 12 junio 2018

Aceptado: 27 noviembre 2018

Publicado on-line: diciembre 2018

\begin{abstract}
The Mediterranean basin is a marine biodiversity hot spot. Despite this, the macroalgal diversity of the Mediterranean Sea is still not fully known, especially in the Mediterranean Marine Protected Areas (MPAs) including, Al-Hoceima National Park of Morocco (PNAH). This paper provides the first comprehensive checklist of the seaweeds of PNAH, based chiefly on our own original collections, and complimented by literature records. Using present-day taxonomy, the total number of taxa at both specific and infraspecific levels currently accepted is 306 taxa with 207 Rhodophyta (39 families), 51 Ochrophyta (13 families) and 48 Chlorophyta (12 families). Ninety five of these species were not found in our samples, 93 were new to the PNAH, and the taxonomic identity of 26 taxa was amended. From the totality of taxa, ten species were reported for the first time from Morocco: 9 Rhodophyta and one green alga. Furthermore, 12 others species (10 red, 1 brown and 1 green alga) are new records for the Mediterranean coast of Morocco. Besides this, confirmed records are mentioned for 20 species, whether in Africa, in Morocco or in the Moroccan Mediterranean coast. This accessible checklist to the international community could serve as an infrastructure for future algal investigations of the taxa in this Specially Protected Area of Mediterranean Importance.
\end{abstract}

Key words: Al-Hoceima National Park, Checklist, Mediterranean Marine Protected Areas, Morocco, Seaweeds.

\section{Resumen}

Lista de algas marinas del Parque Nacional de Alhucemas en Marruecos (Área Marina Protegida del Mediterráneo)

La cuenca del Mediterráneo es un Hot Spot de biodiversidad marina. Aún así, la diversidad de macroalgas del Mediterráneo no está plenamente conocida, especialmente en las Áreas Marinas Protegidas del Mediterráneo (AMP) incluido el Parque Nacional de Alhucemas en Marruecos (PNAH). Este documento proporciona la primera lista exhaustiva de las algas marinas del PNAH. Usando la taxonomía actual, el número total de taxones a niveles específicos y infraespecíficos actualmente aceptados es de 306 taxones con 207 Rhodophyta (39 familias), 51 Ochrophyta (13 familias) y 48 Chlorophyta (12 familias). Noventa y cinco de estas especies no se encontraron en nuestras muestras, 93 eran nuevas en el PNAH y se modificó la identidad taxonómica de 26 taxones. De la totalidad de las macroalgas, 10 especies (9 Rhodophyta y 1 Ulvophyceae) fueron registradas por primera vez desde Marruecos. Además, 12 otras especies (10 Rhodophyta, 1 Phaeophyceae y 1 Ulvophyceae) son nuevos registros para la costa mediterránea de Marruecos. También, se mencionan los registros confirmados de 20 especies, ya sea en África, en Marruecos o en la costa mediterránea marroquí. Esta lista de algas accesibles a la comunidad internacional podría servir como una infraestructura 
para futuras investigaciones algales de los taxones en esta zona especialmente protegida de importancia para el Mediterráneo.

Palabras clave: Parque Nacional de Alhucemas, Lista de algas, Áreas Marinas Protegidas del Mediterráneo, Marruecos, Algas marinas.

\section{Introduction}

The National Park of Al-Hoceima (PNAH) is located in the Alboran Sea in the Southern Mediterranean, Northern Morocco, $150 \mathrm{~km}$ east from the Gibraltar Strait, near to the city of AlHoceima. From the ten National Parks that have a seaboard on the Mediterranean Sea, the PNAH is considered the only National Park that covering a coastline of $40 \mathrm{~km}$. Due to its particular biogeography and important biodiversity, comparable to major Mediterranean Hotspots (Tunesi et al., 2003), the UN classified the PNAH in 2009 as a Specially Protected Area of Mediterranean Importance (PNUE-PAMCAR/ASP, 2009).

The Mediterranean Sea is a region of high biodiversity that ranks among the best known worldwide, and it is considered as a suitable laboratory to study marine ecosystems (Coll et al., 2010). The Mediterranean seaweeds have attracted many botanists (Ballesteros 1990; Ribera et al., 1992; Gómez Garreta et al., 2001; Furnari et al., 2003; Cormaci et al., 2004; Altamirano et al., 2010; Ni-Ni-Win et al., 2011; Tsiamis et al., 2013; Katsanevakis et al., 2014; Verlaque et al., 2015; Cormaci et al., 2012, 2014, 2017; González García et al., 2017). Nevertheless, the knowledge of marine macroalgal diversity of Moroccan Mediterranean coast remains limited by a short history of investigations by some authors (e.g., Conde-Poyales, 1984, 1989; Navarro \& Gallardo, 1989; González-García \& Conde-Poyales, 1991, 1994; González García, 1994; Bueno del Campo \& González García, 1996; Flores Moya \& CondePoyales, 1998). All of these investigations were afterward included as bibliographic references in the single checklists of marine flora of Morocco (Benhissoune et al,. 2001, 2002a, b, 2003). Recently, the combined research efforts of the local phycologists have yielded an important number of new records of seaweeds from the Moroccan coasts and new species to the Mediterranean marine flora (Riadi et al., 2011, 2013; Moussa et al., 2015; Hassoun et al., 2014, 2015, 2016a, b, 2018a, b; Salhi et al., 2016, 2018).

Despite several algal works on the Mediterranean part of Morocco, there is no thorough listing of the seaweeds recorded in the
Specially Protected Mediterranean Area (PNAH). However, it is interesting to signal that the catalogue of marine seaweed conducted by González-García \& Conde-Poyales (1994) includes data from 34 sites spreading on the Moroccan Mediterranean coast, but only two sites among them belong to the Al-Hoceima National Park. Recently, GonzálezGarcía et al. (2017) have published a catalogue of macroalgae of the Peñones de Alhucemas and Vélez de la Gomera (Western Mediterranean, Alboran Sea), and they have reported a total of 107 species in Badis (Peñón de Vélez de la Gomera), the site which localize in the middle of the PNAH.

In light of the previous reasons. In this paper, we attempt to provide a comprehensive database of different macroalgae groups of the PNAH, based chiefly on our own original collections, and complimented by the previous publications. This accessible checklist to the international community would contribute as a serving tool in the Mediterranean marine environmental studies, including prospective research of seaweeds.

\section{Materials and methods}

\section{Study area and time of sampling}

Field collections were performed at seven sites along the $40 \mathrm{~km}$ of coastline of the Al-Hoceima National Park in Morocco (Fig. 1). All specimens were collected by scuba diving down to $15 \mathrm{~m}$ depth and by snorkeling in rocky midlittoral and sublittoral zones (0-2 m depth) throughout the years 20122014 and during each season.

\section{Identification of marine algae}

Seaweeds were transported to the laboratory and stored in $5 \%$ buffered formalin-seawater solution. Species were studied under dissecting or compound microscopes and they were sectioned manually with a razor blade when necessary. Specimens of most of the species included in this checklist are deposited in the Phycological Herbarium of the Phycology and Mycology Group at the Faculty of Sciences, Department of Biology, Abdelmalek Essaâdi University, Tetouan, Morocco. For taxonomic nomenclature purposes, the on-line databases Index Nominum Algarum and AlgaeBase (Guiry \& Guiry, 2018) were used. The checklist 


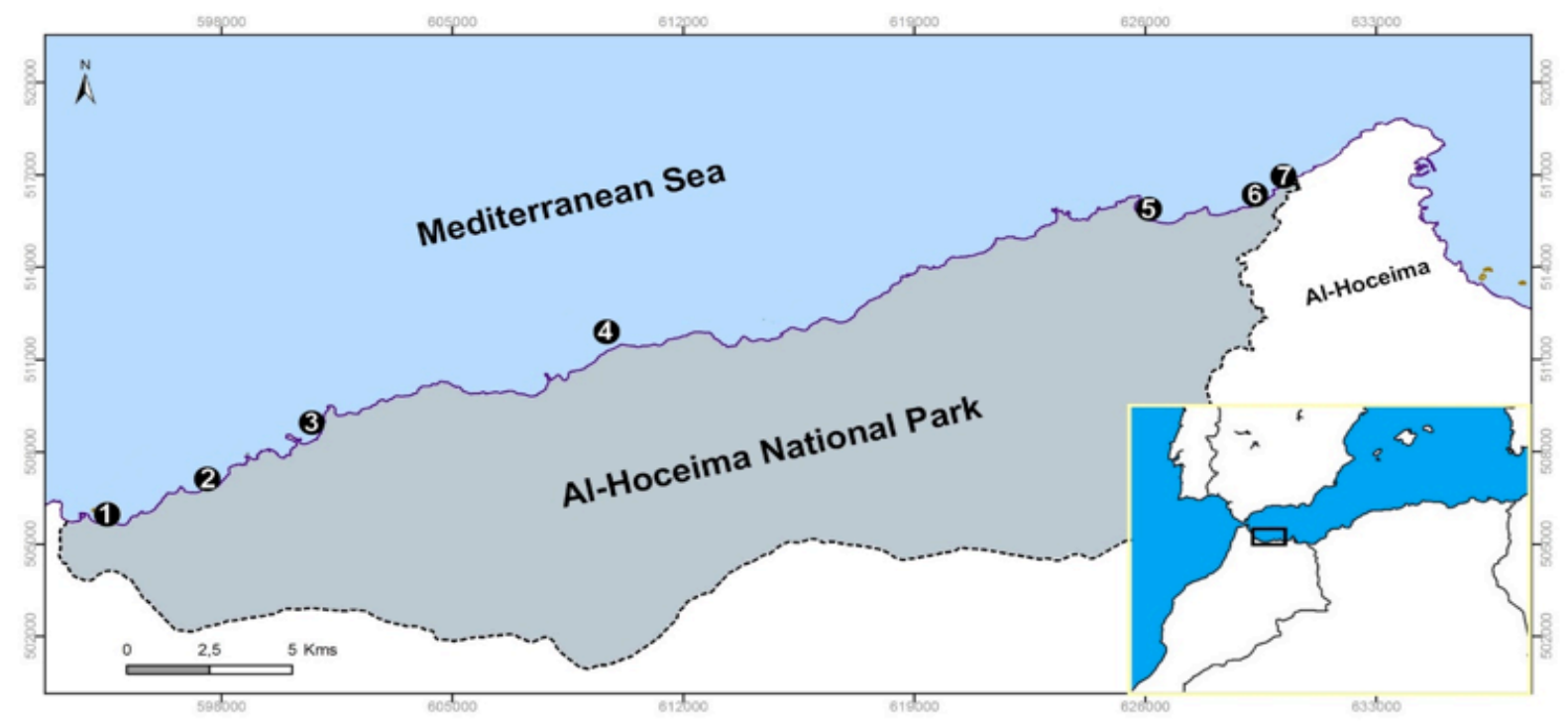

Figure 1. Geographical distribution of the Sampling sites along the Al-Hoceima National Park, northeast of Morocco

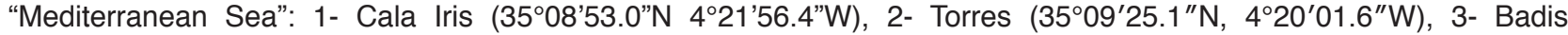

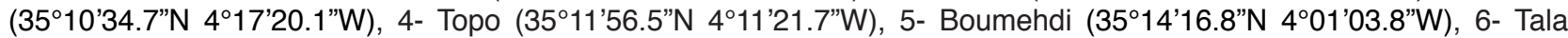
Youssef $\left(35^{\circ} 14^{\prime} 11.7^{\prime \prime} \mathrm{N} 3^{\circ} 58^{\prime} 55.5^{\prime \prime} \mathrm{W}\right), 7-\mathrm{Rmoud}\left(35^{\circ} 14^{\prime} 41.5^{\prime \prime} \mathrm{N}, 3^{\circ} 57^{\prime} 55.7^{\prime \prime} \mathrm{W}\right)$.

Figura 1: Distribución geográfica de los sitios de muestreo a lo largo del Parque Nacional de Alhucemas, al noreste

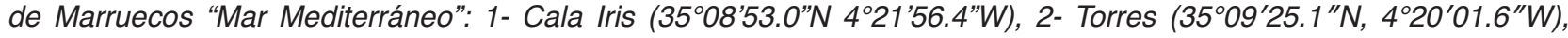

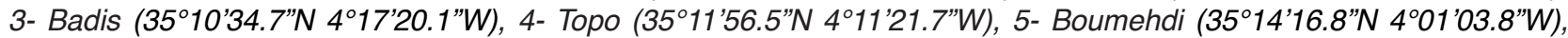

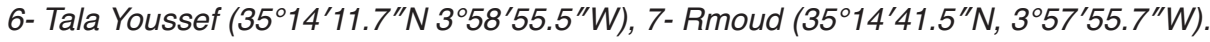

of marine macroalgae of PNAH was compiled of personal collections in this study and two previous literatures data: González-García \& CondePoyales (1994) and González-García et al. (2017). The previously known species, together with the new species from PNAH and Morocco were mainly verified using AlgaeBase and after a careful review of all published records including, the checklists of seaweeds of Morocco (Benhissoune et al., 2001, 2002a, b, 2003). On the other side, unpublished works such as thesis, master dissertations and technical reports have not been considered owing to the lack a scrutinized in their elaboration.

\section{Results and discussion}

The checklist presented in this work includes 306 taxa at specific and intraspecific level, including 207 Rhodophyta, 51 Ochrophyta, and 48 Chlorophyta (List of taxa: Accepted seaweed taxa hitherto reported in the Al-Hoceima National Park according to their sampling site, based on both our collections and previous records by González-García \& Conde-Poyales (1994) and González-García et al. (2017). Principally, 211 taxa (144 Rhodophyta, 30 Phaeophyceae and
37 Ulvophyceae) were identified from all of our sampling expeditions. Among our collections, 10 species are new for Morocco and 12 others species are new records for the Mediterranean cost of Morocco.

In the catalogue, taxa of marine macroalgae are arranged alphabetically in three major groups (Chlorophyta, Ochrophyta and Rhodophyta), and following the classification systems and the nomenclature presented in AlgaeBase (Guiry \& Guiry, 2018). The species locations in the current study are given (1-7) and previous reference records are also provided (González-García \& Conde-Poyales, 1994 and González-García et al., 2017).

The sites are as follows: Cala Iris (1); Torres (2); Badis (3); Topo (4); Boumehdi (5); Tala Youssef (6) and Rmod (7). Confirmed presence in previous study (P1: González-García \& CondePoyales (1994), P2: González-García et al. (2017). Superscript number refer to the Notes. Authors of taxa are given in full, and synonyms of amended taxa are also given. New records for Morocco are marked with an asterisk [ $\left.{ }^{*}\right]$ before the name of a taxon. 


\section{LIST OF TAXA}

Taxa

Phylum Chlorophyta

\section{Class Ulvophyceae}

Order Bryopsidales

\section{Family Bryopsidaceae}

Bryopsidella neglecta (Berthold) G. Furnari \& M. Cormaci

${ }^{\star}$ Bryopsis cupressina J.V. Lamouroux

Bryopsis duplex De Notaris

Bryopsis hypnoides J.V. Lamouroux

Bryopsis muscosa J.V. Lamouroux

Bryopsis plumosa (Hudson) C. Agardh

Family Codiaceae

Codium adhaerens C. Agardh

Codium decorticatum (Woodward) M. Howe

Codium effusum (Rafinesque) Delle Chiaje

Codium tomentosum Stackhouse

\section{Family Derbesiaceae}

Derbesia tenuissima (Moris \& De Notaris) P.L. Crouan \& H.M. Crouan

\section{Family Udoteaceae}

Pseudochlorodesmis furcellata (Zanardini) Børgesen ${ }^{9}$

\section{Order Cladophorales}

Family Boodleaceae

Cladophoropsis membranacea (Hofman Bang ex C. Agardh) Børgesen ${ }^{1}$

\section{Family Cladophoraceae}

Chaetomorpha aerea (Dillwyn) Kützing

Chaetomorpha ligustica (Kützing) Kützing

Chaetomorpha linum (O.F. Müller) Kützing

Cladophora albida (Nees) Kutzing

Cladophora coelothrix Kützing

Cladophora dalmatica Kützing

Cladophora hutchinsiae (Dillwyn) Kützing

Cladophora laetevirens (Dillwyn) Kützing

Cladophora lehmanniana (Lindenberg) Kützing

Cladophora prolifera (Roth) Kützing

Cladophora rupestris (Linnaeus) Kützing

\section{Location in this study}

Previous reference

\section{$1,2,5,6,7$}

1

$1,6,7$

$1,4,6$

$1,2,3,5,6,7$

1,7

1,4

$P_{1}, P_{2}$

$\mathrm{P}_{2}$

$\mathrm{P}_{1}, \mathrm{P}_{2}$
$1,6,7$

6,7

2, 5, 6, 7

$1,2,5,7$

$1,5,6,7$

$\mathrm{P}_{1}, \mathrm{P}_{2}$

1,7

$P_{1}$

$1,6,7$

$\mathrm{P}_{1}$

1,7

1, 2, 4, 5, 6, $7 \quad P_{1}$

1,7

$P_{1}$

$\mathrm{P}_{1}$ 
Taxa

Cladophora sericea (Hudson) Kützing

Cladophora socialis Kützing ${ }^{8}$

Cladophora vagabunda (Linnaeus) Hoek

Lychaete battersii (C.Hoek) M.J. Wynne ${ }^{8}$

Lychaete echinus (Biasoletto) M.J. Wynne = Cladophora echinus (Biasoletto) Kützing

Rhizoclonium tortuosum (Dillwyn) Kützing

\section{Family Siphonocladaceae}

Siphonocladus tropicus (P. Crouan \& H. Crouan) J. Agardh

Family Valoniaceae

Valonia macrophysa Kützing

Valonia utricularis (Roth) C. Agardh

\section{Order Ulvales}

\section{Family Kornmanniaceae}

Blidingia marginata (J. Agardh) P.J.L. Dangeard ex Bliding

Blidingia minima (Nägeli ex Kützing) Kylin

Family Phaeophilaceae

Phaeophila dendroides (P. Crouan \& H. Crouan) Batters

\section{Order Ulotrichales}

\section{Family Ulotrichaceae}

Ulothrix flacca (Dillwyn) Thuret

\section{Family Ulvaceae}

Ulva clathrata (Roth) C. Agardh

Ulva compressa Linnaeus

Ulva flexuosa Wulfen

Ulva intestinalis Linnaeus

= Enteromorpha intestinalis (Linnaeus) Nees

Ulva lactuca Linnaeus

Ulva linza Linnaeus

Ulva polyclada Kraft

Ulva prolifera O.F. Müller

$=$ Enteromorpha prolifera (O.F.Müller) J.Agardh

Ulva rigida C. Agardh

Ulva torta (Mertens) Trevisan

\section{Family Ulvellaceae}

Ulvella viridis (Reinke) R. Nielsen, C.J. O'Kelly \& B. Wysor $=$ Entocladia viridis Reinke

\section{Phylum Ochrophyta}

\section{Class Phaeophyceae}

Location in this study

1

$1,2,7$

1

$P_{1}, P_{2}$

3, 7

$$
\begin{aligned}
& P_{1} \\
& P_{1}, P_{2}
\end{aligned}
$$

1

6

$1,6,7 \quad P_{1}$

1

$\mathrm{P}_{1}$

$\mathrm{P}_{2}$

7

$P_{1}$

$1,4,6,7$

$\mathrm{P}_{2}$

$1,2,3,6,7$

$\mathrm{P}_{2}$

$1,4,6,7$

$\mathrm{P}_{1}$

$\mathrm{P}_{1}$

$1,2,3,4,5,6,7$

1

$\mathrm{P}_{1}, \mathrm{P}_{2}$

$\mathrm{P}_{1}, \mathrm{P}_{2}$

1

$\mathrm{P}_{1}$

$1,2,5,6,7$

$\mathrm{P}_{1}, \mathrm{P}_{2}$

$\mathrm{P}_{2}$

1, 7

$P_{1}$ 
Order Dictyotales

Family Dictyptaceae

Dictyopteris polypodioides (A.P. De Candolle) J.V.

6,7

Dictyota dichotoma var. intricata (C. Agardh) Greville

Dictyota fasciola (Roth) J.V. Lamouroux

Dictyota spiralis Montagne

Padina pavonica (Linnaeus) Thivy

Spatoglossum solieri (Chauvin ex Montagne) Kützing Taonia atomaria (Woodward) J. Agardh

Zonaria tournefortii (J.V. Lamouroux) Montagne

\section{Order Ectocarpales}

\section{Family Acinetosporaceae}

Feldmannia irregularis (Kützing) Hamel

Feldmannia lebelii (Areschoug ex P. Crouan \& H. Crouan)

Hamel

Feldmannia mitchelliae (Harvey) H.-S. Kim

Feldmannia simplex (P. Crouan \& H. Crouan) Hamel ${ }^{9}$

Hincksia hincksiae (Harvey) P.C. Silva

\section{Family Ectocarpaceae}

Ectocarpus crouanii Thuret ${ }^{5}$

Ectocarpus fasciculatus Harvey

Ectocarpus siliculosus (Dillwyn) Lyngbye

Family Scytosiphonaceae

Colpomenia peregrina Sauvageau

Colpomenia sinuosa (Mertens ex Roth) Derbès \& Solier

Petalonia fascia (O.F. Müller) Kuntze

Scytosiphon lomentaria (Lyngbye) Link

\section{Order Fucales}

\section{Family Fucaceae}

Fucus spiralis Linnaeus

Fucus vesiculosus Linnaeus

\section{Family Sargassaceae}

Cystoseira brachycarpa J. Agardh

Cystoseira compressa (Esper) Gerloff \& Nizamuddin

Cystoseira elegans Sauvageau

Cystoseira humilis Schousboe ex Kützing

Cystoseira mauritanica Sauvageau
$1,2,4,5,6,7$

7
1

$1,2,3$

$P_{1}, P_{2}$

1

1

1

1,7

$\begin{array}{ll}1,2,4,5,6,7 & \mathrm{P}_{1}, \mathrm{P}_{2} \\ 6 & \mathrm{P}_{1} \\ 6 & \mathrm{P}_{1}, \mathrm{P}_{2}\end{array}$

$P_{1}$

$$
P_{1}
$$

$\mathrm{P}_{2}$

$\mathrm{P}_{1}, \mathrm{P}_{2}$

$P$

${ }_{1}, \mathrm{P}_{2}$

$$
\begin{aligned}
& P_{1}, P_{2} \\
& P_{1} \\
& P_{1} \\
& P_{1} \\
& P_{1}, P_{2} \\
& P_{1} \\
& P_{1} \\
& P_{1}
\end{aligned}
$$

$P_{1}$ 1

$1,4,5,6,7$

$\mathrm{P}_{1}$ $\mathrm{P}_{1}, \mathrm{P}_{2}$ $\mathrm{P}_{1}$ $P_{1}$ $\mathrm{P}_{1}$ 
Taxa

Cystoseira mediterranea Sauvageau

Cystoseira montagnei J.Agardh

= Cystoseira spinosa Sauvageau

Cystoseira nodicaulis (Withering) M.Roberts

Cystoseira sauvageauana Hamel

Cystoseira spinosa Sauvageau

Cystoseira tamariscifolia (Hudson) Papenfuss

Cystoseira usneoides (Linnaeus) M. Roberts

Sargassum hornschuchii C. Agardh

Sargassum vulgare C. Agardh

\section{Order Ralfsiales}

\section{Family Hapalospongidiaceae}

Hapalospongidion macrocarpum (Feldmann) León-Álvarez \& González-González

Ralfsia verrucosa (Areschoug) Areschoug

\section{Order Sphacelariales}

\section{Family Cladostephaceae}

Cladostephus spongiosum (Hudson) C. Agardh

Cladostephus spongiosum f. verticillatum (Lightf.)

Prud'homme van Reine

\section{Family Lithodermataceae}

Pseudolithoderma adriaticum (Hauck) Verlaque ${ }^{1}$

\section{Family Sphacelariaceae}

Sphacelaria cirrosa (Roth) C. Agardh

Sphacelaria fusca (Hudson) S.F. Gray

Sphacelaria plumula Zanardini

Sphacelaria rigidula Kützing

Sphacelaria tribuloides Meneghini

Family Stypocaulaceae

Halopteris filicina (Grateloup) Kützing

Halopteris scoparia (Linnaeus) Sauvageau

Order Tilopteridales

Family Phyllariaceae

Phyllariopsis brevipes (C. Agardh) E.C. Henry \& G.R. South

Saccorhiza polyschides (Lightfoot) Batters
Location in this study

Previous reference

6,7

$\begin{array}{ll} & P_{2} \\ & P_{2} \\ & P_{1} \\ & P_{2} \\ 1,2,4,5,6,7 & P_{1}, P_{2} \\ & P_{1} \\ 1,3,6,7 & P_{1} \\ & P_{1}, P_{2}\end{array}$

$1,3,4,5,7$

$\mathrm{P}_{1}$

$\begin{array}{ll}1,5,6,7 & \mathrm{P}_{1} \\ 3,7 & \mathrm{P}_{1} \\ 1,3,7 & \mathrm{P}_{1}, \mathrm{P}_{2} \\ 1,4,7 & \mathrm{P}_{1}, \mathrm{P}_{2} \\ 1,5,7 & \mathrm{P}_{2} \\ 1,7 & \\ 1,2,3,5,6,7 & \mathrm{P}_{1}, \mathrm{P}_{2} \\ & \mathrm{P}_{1}, \mathrm{P}_{2}\end{array}$

$1,5,6,7$

$P_{1}$

${ }_{1}, P_{2}$

$P_{1}, P_{2}$

$\mathrm{P}_{2}$

$\mathrm{P}_{1}$

\section{Phylum Rhodophyta}

\section{Class Bangiophyceae}

Order Bangiales 
Taxa

Family Bangiaceae

Bangia atropurpurea (Mertens ex Roth) C. Agardh

Bangia fuscopurpurea (Dillwyn) Lyngbye

Porphyra umbilicalis Kützing

Pyropia leucosticta (Thuret) Neefus \& J. Brodie

\section{Class Compsopogonophyceae}

Order Erythropeltales

Family Erythrotrichiaceae

${ }^{\star}$ Erythrotrichia bertholdii Batters

Erythrotrichia carnea (Dillwyn) J. Agardh

Erythrotrichia investiens (Zanardini) Bornet ${ }^{6}$

Erytrotrichia reflexa (P.L. Crouan et H.M. Crouan) Thuret ex De Toni

Porphyrostromium ciliare (Carmichael) M.J. Wynne ${ }^{1,7}$

\section{Class Florideophyceae}

\section{Order Acrochaetiales}

\section{Family Acrochaetiaceae}

Acrochaetium microscopicum (Nägeli ex Kützing) Nägeli

Acrochaetium parvulum (Kylin) Hoyt

Acrochaetium trifilum (Buffham) Batters

Grania efflorescens (J. Agardh) Kylin

$$
\text { = Acrochaetium thuretii (Bornet) Collins \& Hervey }
$$

\section{Order Bonnemaisoniales}

\section{Family Bonnemaisoniaceae}

Asparagopsis armata Harvey

Asparagopsis taxiformis (Delile) Trevisan ${ }^{8}$

Bonnemaisonia hamifera Hariot

\section{Order Ceramiales}

\section{Family Callithamniaceae}

Aglaothamnion cordatum Feldmann-Mazoyer

Aglaothamnion tenuissimum (Bonnemaison) FeldmannMazoyer

=Aglaothamnion byssoides (Arnott ex Harvey) C.F. Boudouresque \& M.M.Perret-Boudouresque

Callithamnion corymbosum (Smith) Lyngbye

Callithamnion granulatum (Ducluzeau) C. Agardh

Callithamnion tetragonum (Withering) S.F. Gray

Callithamnion tetricum (Dillwyn) S.F.Gray ${ }^{9}$

Gaillona gallica (Nägeli) Athanasiadis

= Aglaothamnion gallicum (Nägeli) L'Hardy-Halos ex F. Ardré

\section{Location in this study}

\section{Previous reference}

1,7

$1,2,4,6,7$

1,7
$\mathrm{P}_{2}$

$\mathrm{P}_{2}$

$1,2,6,7$

$\mathrm{P}_{2}$

$\mathrm{P}_{1}, \mathrm{P}_{2}$

$1,2,4,5,6,7$

$\mathrm{P}_{1}, \mathrm{P}_{2}$

1, 3, 4, 5, 6, 7

1, 7

1,4

1,6

$\mathrm{P}_{1}$

1, 2, 3, 5, 6, $7 \quad \mathrm{P}_{1}, \mathrm{P}_{2}$

1, 6, $7 \quad P_{1}$

1, 3, 6, $7 \quad P_{1}$

$1,2,4,6,7$

$\mathrm{P}_{1}, \mathrm{P}_{2}$

$\mathrm{P}_{1}, \mathrm{P}_{2}$

$\mathrm{P}_{1}$

$\mathrm{P}_{1}$ 
Taxa

Gaillona hookeri (Dillwyn) Athanasiadis

= Aglaothamniom hookeri (Dillwyn) C.M. Maggs et M.H. Hommersand

Gaillona scopulorum (C. Agardh) Athanasiadis ${ }^{9}$

Gayliella flaccida (Harvey ex Kützing) T.O. Cho \& L.J. Mclvor

Gayliella taylorii (E.Y. Dawson) T.O. Cho \& S. M.Boo ${ }^{8}$

Seirospora giraudyi (Kützing) De Toni

\section{Family Ceramiaceae}

Antithamnion amphigeneum A. Millar

= Antithamnion algeriense M.Verlaque \& Seridi

Antithamnion cruciatum (C. Agardh) Nägeli

${ }^{\star}$ Antithamnion decipiens (J. Agardh) Athanasiadis

*Antithamnionella boergesenii (Cormaci \& G. Furnari)

Athanasiadis

Antithamnionella elegans (Berthold) J.H. Price \& D. M.John

Antithamnionella spirographidis (Schiffner) E.M. Wollaston

Centroceras gasparrinii (Meneghini) Kützing ${ }^{4}$

Ceramium ciliatum (J. Ellis) Ducluzeau var. ciliatum

Ceramium ciliatum var. robustum (J. Agardh) Mazoyer

Ceramium circinatum (Kützing) J. Agardh

Ceramium codii (H. Richards) Mazoyer

Ceramium diaphanum (Lightfoot) Roth

Ceramium echionotum J. Agardh

Ceramium giacconei Cormaci \& G.Furnari ${ }^{2}$

Ceramium secundatum Lyngbye

Ceramium siliquosum var. lophophorum (Feldman-Mazoyer)

Serio

=Ceramium diaphanum var. lophophorum Feldmann-

Mazoyer

Ceramium tenerrimum (G. Martens) Okamura

Ceramium virgatum Roth var. virgatum ${ }^{3}$

Ceramium virgatum var. implexocontortum (Solier) G. Furnari

Compsothamnion thuioides (Smith) Nägeli

Crouania attenuata (C. Agardh) J. Agardh

Pleonosporium borreri (Smith) Nägeli

Pterothamnion crispum (Ducluzeau) Nägeli

Pterothamnion plumula (J. Ellis) Nägeli

Spongoclonium caribaeum (Børgesen) M.J. Wynne ${ }^{8}$

\section{Family Dasyaceae}

Dasya corymbifera J. Agardh ${ }^{1}$
Location in this study

\section{Previous reference}

$$
\mathrm{P}_{1}, \mathrm{P}_{2}
$$

2

4

$$
\mathrm{P}_{1}, \mathrm{P}_{2}
$$

1, 2, 3, 5, 7

$P_{1}$

$P_{1}$

7

$\mathrm{P}_{1}, \mathrm{P}_{2}$

1

7

1

1, 2, 3, 5, 6, 7

1, 2, 3, 4, 5, 6, 7

$P_{1}, P_{2}$

$\mathrm{P}_{1}$

$\mathrm{P}_{1}$

$1,4,7$

1, 2, 3, 4, 6, $7 \quad \mathrm{P}_{1}, \mathrm{P}_{2}$

$1,4,6,7$

$\mathrm{P}_{1}, \mathrm{P}_{2}$

1,4

$5,6,7$

$\mathrm{P}_{2}$

$\mathrm{P}_{1}$

$\mathrm{P}_{1}, \mathrm{P}_{2}$

$1,2,5,6,7$

$1,5,6,7$

$P$

1, 2, 6, 7

$\mathrm{P}_{1}$

$1,4,7$

$\mathrm{P}_{1}$

4, 7

$P_{1}$

4

$1,2,4,6$ 


\section{Taxa}

Dasya hutchinsiae Harvey

Dasya ocellata (Grateloup) Harvey

Dasya rigidula (Kützing) Ardissone

\section{Family Delesseriaceae}

Acrosorium ciliolatum (Harvey) Kylin

= Acrosorium venulosum (Zanardini) Kylin

Apoglossum ruscifolium (Turner) J. Agardh

Cryptopleura ramosa (Hudson) L. Newton

Myriogramme minuta Kylin

Nitophyllum punctatum (Stackhouse) Greville ${ }^{9}$

Radicilingua thysanorhizans (Holmes) Papenfuss

Taenioma nanum (Kützing) Papenfuss

\section{Family Rhodomelaceae}

Alsidium corallinum $\mathrm{C}$. Agardh

Aphanocladia stichidiosa (Funk) Ardré

Chondria capillaris (Hudson) M.J. Wynne

Chondria coerulescens (J. Agardh) Sauvageau

Chondria dasyphylla (Woodward) C. Agardh

Chondria mairei G. Feldmann

Chondrophycus undulatus (Yamada) Garbary \& Harper

Halopithys incurva (Hudson) Batters

Herposiphonia secunda (C. Agardh) Ambronn

Herposiphonia secunda f. tenella (C. Agardh) M.J. Wynne

Herposiphonia tenella (C. Agardh) Ambronn

Heterosiphonia crispella (C. Agardh) M.J. Wynne

Hypoglossum hypoglossoides (Stackhouse) Collins \& Hervey

Laurencia obtusa (Hudson) J.V. Lamouroux

*Melanothamnus harveyi (Bailey) Díaz-Tapia \& Maggs

*Melanothamnus sphaerocarpus (Børgesen) Díaz-Tapia \& Maggs

Osmundea pinnatifida (Hudson) Stackhouse

Palisada perforata (Bory) K.W. Nam

= Laurencia papillosa (C.Agardh) Greville

Polysiphonia atlantica Kapraun \& J.N. Norris

Polysiphonia brodiei (Dillwyn) Sprengel

Polysiphonia denudata (Dillwyn) Greville ex Harvey

Polysiphonia dichotoma Kützing

Polysiphonia elongata (Hudson) Sprengel

\section{Location in this study}

$1,4,7$

7

$1,2,5,7$

$1,3,4,6,7$

$P_{1}$

4

1,7

$\mathrm{P}_{1}, \mathrm{P}_{2}$

$P_{1}$

$\mathrm{P}_{1}, \mathrm{P}_{2}$

1,2

$1,2,5,7$

$1,4,6,7$

$\mathrm{P}_{2}$

$1,2,5,6,7$

$\mathrm{P}_{1}, \mathrm{P}_{2}$

1, 2, 6,7

$\mathrm{P}_{1}$

$P_{1}$

$1,2,3,6,7$

$\mathrm{P}_{1}, \mathrm{P}_{2}$

$\mathrm{P}_{1}$

$\mathrm{P}_{1}$

6, 7

$\mathrm{P}_{1}$

$1,2,3,5,6,7$

$\mathrm{P}_{1}, \mathrm{P}_{2}$

$P_{1}$

$1,2,4,5,6$

$1,6,7$

$\mathrm{P}_{1}, \mathrm{P}_{2}$

1, 7

$\mathrm{P}_{1}, \mathrm{P}_{2}$

6, 7

$\mathrm{P}_{1}, \mathrm{P}_{2}$

7

1, 4, 7

$1,2,3,6,7$

$\mathrm{P}_{1}, \mathrm{P}_{2}$

$\mathrm{P}_{1}$

7

6

1, 4, 7

$\mathrm{P}_{1}$

$P_{1}$

$\mathrm{P}_{1}$ 
Taxa

Polysiphonia fibrillosa (Dillwyn) Sprengel

${ }^{\star}$ Polysiphonia flocculosa (C. Agardh) Endlichter

Polysiphonia havanensis Montagne

Polysiphonia opaca (C. Agardh) Moris \& De Notaris

Polysiphonia sertularioides (Grateloup) J. Agardh

Polysiphonia stricta (Mertens ex Dillwyn) Greville ${ }^{5}$

Pterosiphonia complanata (Clemente) Falkenberg

Rytiphlaea tinctoria (Clemente) C. Agardh

Symphyocladia parasitica (Hudson) Savoie \& G.W. Saunders

= Pterosiphonia parasitica (Hudson) Falkenberg

Vertebrata fruticulosa (Wulfen) Kuntze

= Boergeseniella fruticulosa (Wulfen) Kylin

Vertebrata fucoides (Hudson) Kuntze

= Polysiphonia fucoides (Hudson) Greville

Vertebrata reptabunda (Suhr) Díaz-Tapia \& Maggs

$=$ Lophosiphonia reptabunda (Suhr) Kylin

Vertebrata thuyoides (Harvey) Kuntze

Vertebrata tripinnata (Harvey) Kuntze

Xiphosiphonia pennata (C. Agardh) Savoie \& G.W. Saunders

Family Sarcomeniaceae

Cottoniella filamentosa (M. Howe) Børgesen

\section{Family Spyridiaceae}

Spyridia filamentosa (Wulfen) Harvey

Family Wrangeliaceae

Diplothamnion jolyi C. Hoek

Griffithsia opuntioides J. Agardh

Halurus flosculosus (J. Ellis) Maggs \& Hommersand

Lejolisia mediterranea Bornet

Monosporus pedicellatus (Smith) Solier

Spermothamnion flabellatum Bornet

Spermothamnion repens (Dillwyn) Magnus ${ }^{9}$

Tiffaniella capitata (Bornet) Doty \& Meñez ${ }^{1}$

Tiffaniella gorgonea (Montagne) Doty \& Meñez 1960

\section{Order Colaconematales}

Family Colaconemataceae

Colaconema caespitosum (J. Agardh) Jackelman, Stegenga

\& J.J. Bolton

Colaconema daviesii (Dillwyn) Stegenga
Location in this study

\section{Previous reference}

6

1

6,7

$1,6,7$

$P_{1}, P_{2}$

3,6

$\mathrm{P}_{1}, \mathrm{P}_{2}$

6,7

$1,2,3,5,6,7 \quad \mathrm{P}_{1}, \mathrm{P}_{2}$

7

$\mathrm{P}_{1}$

$1,2,5,6,7 \quad P_{1}, P_{2}$

$P_{1}$

$P_{1}$

$1,6,7 \quad \mathrm{P}_{1}, \mathrm{P}_{2}$

1,7

7

$P_{1}, P_{2}$

$\mathrm{P}_{2}$

$\begin{array}{ll}1,7 & P_{1}\end{array}$

1,7

$1,4,6,7 \quad P_{1}$

$P_{1}$

$\mathrm{P}_{1}$

1,6

$P_{1}$

7

5

5, 7

1

$1,4 \quad \mathrm{P}_{1}, \mathrm{P}_{2}$ 
Taxa

Colaconema savianm (Meneghini) R. Nielsen

= Acrochaetium savianum (Meneghini) Nägeli

\section{Order Corallinales}

\section{Family Corallinaceae}

Amphiroa beauvoisii J.V. Lamouroux

Amphiroa cryptarthrodia Zanardini

Amphiroa fragilissima (Linnaeus) J.V. Lamouroux

Amphiroa rigida J.V. Lamouroux

Corallina officinalis Linnaeus

Ellisolandia elongata (J. Ellis \& Solander) K.R. Hind \& G.W. Saunders

Hydrolithon farinosum (J.V. Lamouroux) Penrose \& Y.M.

Chamberlain

= Fosliella farinosa (J.V.Lamouroux) M.Howe

* Jania capillacea Harvey

Jania longifurca Zanardini

Jania rubens (Linnaeus) J.V. Lamouroux

Jania rubens var. corniculata (Linnaeus) Yendo

= Jania corniculata (Linnaeus) J.V.Lamouroux

Jania squamata (Linnaeus) J.H. Kim, Guiry \& H.-G. Choi ${ }^{9}$

Jania virgata (Zanardini) Montagne

$=$ Corallina granifera J.Ellis \& Solander

Lithophyllum byssoides (Lamarck) Foslie

Lithophyllum corallinae (P. Crouan \& H. Crouan) Heydrich ${ }^{1}$

Lithophyllum cystoseirae (Hauck) Heydrich

= Titanoderma cystoseirae (Hauck) Woelkerling,

Y.M.Chamberlain \& P.C.Silva

Lithophyllum dentatum (Kützing) Foslie

= Spongites dentatus Kützing

Lithophyllum incrustans Philippi

Neogoniolithon brassica-florida (Harvey) Setchell \& L.R.

Mason

Pneophyllum confervicola (Kützing) Y.M. Chamberlain ${ }^{8}$

Pneophyllum fragile Kützing

= Pneophyllum lejolisii (Rosanoff) Y.M.Chamberlain

Tenarea tortuosa (Esper) Me. Lemoine

Titanoderma pustulatum (J.V. Lamouroux) Nägeli

Family Lithothamniaceae

Phymatolithon lenormandii (Areschoug) W.H.Adey

Order Gelidiales

Family Gelidiaceae

\section{Location in this study}

\section{Previous reference}

$P_{1}$

4,7

$P_{1}$

$\mathrm{P}_{1}$

1

7

$P_{1}$

$1,2,3,5,6,7$

$P_{1}$

$1,2,3,4,5,6,7$

$\mathrm{P}_{1}, \mathrm{P}_{2}$

$P_{1}$

6

$1,2,4,5,6,7$

$\mathrm{P}_{1}, \mathrm{P}_{2}$

$1,2,3,4,5,7$

$P_{1}, P_{2}$

$\mathrm{P}_{1}$

$1,3,5,7$

$\mathrm{P}_{1}$

$\mathrm{P}_{2}$

6

$P_{1}$

7

$P_{1}$

$1,4,6,7$

$P_{1}, P_{2}$

$\mathrm{P}_{1}, \mathrm{P}_{2}$

1,7

$P_{1}$

$1,6,7$

$1,2,3,5,6,7 \quad \mathrm{P}_{1}, \mathrm{P}_{2}$ 


\section{Taxa}

Gelidiella lubrica (Kützing) Feldmann \& Hamel

Gelidium attenuatum (Turner) Thuret

Gelidium bipectinatum $\mathrm{G}$. Furnari ${ }^{1}$

Gelidium corneum (Hudson) J.V. Lamouroux

Gelidium crinale (Hare ex Turner) Gaillon

Gelidium microdon Kützing

Gelidium minusculum (Weber-van Bosse) R.E. Norris

Gelidium pulchellum (Turner) Kützing

Gelidium pusillum (Stackhouse) Le Jolis var. pusillum

Gelidium pusillum var. pulvinatum (C. Agardh) Feldmann

Gelidium spathulatum (Kützing) Bornet

Gelidium spinosum (S.G. Gmelin) P.C. Silva var. spinosum

Gelidium spinosum var. hystrix (J. Agardh) G. Furnari

$=$ Gelidium latifolium var. hystrix (J.Agardh) Hauck

\section{Family Pterocladiaceae}

Pterocladiella capillacea (S.G. Gmelin) Santelices \& Hommersand

Pterocladiella melanoidea (Schousboe ex Bornet) Santelices \& Hommersand

\section{Order Gigartinales}

\section{Family Caulacanthaceae}

Caulacanthus ustulatus (Mertens ex Turner) Kützing

\section{Family Cystocloniaceae}

Hypnea musciformis (Wulfen) J.V. Lamouroux

Hypnea spinella (C. Agardh) Kützing ${ }^{1}$

${ }^{\star}$ Hypnea valentiae (Turner) Montagne

Rhodophyllis divaricata (Stackhouse) Papenfuss ${ }^{1}$

\section{Family Gigartinaceae}

Chondracanthus acicularis (Roth) Fredericq

Chondracanthus teedei (Mertens ex Roth) Kützing

Gigartina pistillata (S.G. Gmelin) Stackhouse

\section{Family Phyllophoraceae}

Gymnogongrus crenulatus (Turner) J. Agardh

Gymnogongrus griffithsiae (Turner) Martius

Gymnogongrus patens (Goodenough \& Woodward) J. Agardh

Phyllophora crispa (Hudson) P.S. Dixon

Phyllophora sicula (Kützing) Guiry \& L.M. Irvine

Schottera nicaeensis (J.V. Lamouroux ex Duby) Guiry \& Hollenberg

\section{Location in this study}

\section{Previous reference}

1, 7

3, 6, 7

1

7

$P_{1}, P_{2}$

$2,5,6,7$

$\mathrm{P}_{1}, \mathrm{P}_{2}$

1

1, 7

1, 6, 7

1, 6, 7

$\mathrm{P}_{1}, \mathrm{P}_{2}$

1, 2, 7

1

$\mathrm{P}_{1}, \mathrm{P}_{2}$

$1,2,6,7$

$\mathrm{P}_{1}, \mathrm{P}_{2}$

$\mathrm{P}_{1}$

$1,6,7 \quad \mathrm{P}_{1}, \mathrm{P}_{2}$

7
1, 7
$\mathrm{P}_{2}$

1, 3, 5, 6, $7 \quad \mathrm{P}_{1}, \mathrm{P}_{2}$

5,7

7

1, 6, 7

1, 2, 3, 4, 6, $7 \quad \mathrm{P}_{1}, \mathrm{P}_{2}$

7

6, 7

$\mathrm{P}_{1}, \mathrm{P}_{2}$

$P_{1}, P_{2}$

6, 7

1, 3, 6, 7 


\section{Taxa}

Stenogramma interruptum (C. Agardh) Montagne ${ }^{9}$

Family Rissoellaceae

Rissoella verruculosa (Bertoloni) J. Agardh

Family Schmitziellaceae

Schmitziella endophloea Bornet \& Batters

Family Sphaerococcaceae

Sphaerococcus coronopifolius Stackhouse

Order Gracilariales

Family Gracilariaceae

Gracilaria armata (C. Agardh) Greville

Gracilaria bursa-pastoris (S.G. Gmelin) P.C. Silva

Gracilaria conferta (Schousboe ex Montagne) Montagne ${ }^{1}$

Gracilaria dura (C. Agardh) J. Agardh ${ }^{1}$

Gracilaria multipartita (Clemente) Harvey

Gracilariopsis longissima (S.G. Gmelin) M. Steentoft, L.M. Irvine \& W.F. Farnham

\section{Order Halymeniales}

\section{Family Halymeniaceae}

Grateloupia filicina (J.V. Lamouroux) C. Agardh

Order Hapalidiales

\section{Family Hapalidiaceae}

Choreonema thuretii (Bornet) F. Schmitz

Melobesia membranacea (Esper) J.V. Lamouroux

Family Mesophyllaceae

Mesophyllum expansum (Philippi) Cabioch \& M.L. Mendoza $=$ Lithophyllum expansum Philippi

Mesophyllum lichenoides (J.Ellis) Me. Lemoine

Order Hildenbrandiales

Family Hildenbrandiaceae

Hildenbrandia rubra (Sommerfelt) Meneguini

Order Nemaliales

\section{Family Liagoraceae}

Liagora distenta (Mertens ex Roth) J.V. Lamouroux

Liagora viscida (Forsskål) C. Agardh

Family Nemaliaceae

Nemalion elminthoides (Velley) Batters

Family Scinaiaceae

\section{Location in this study}

6

1,2

$P_{1}$

$$
\mathrm{P}_{1}, \mathrm{P}_{2}
$$

$1,4,7$

$$
\mathrm{P}_{1}, \mathrm{P}_{2}
$$

$\mathrm{P}_{1}$

$P_{1}, P_{2}$

7

7

6

3, 7
$P_{1}$

$P_{1}$

$\mathrm{P}_{1}, \mathrm{P}_{2}$

$P_{1}$

$\mathrm{P}_{1}, \mathrm{P}_{2}$
$P_{1}$

$\mathrm{P}_{1}$

6,7
$\mathrm{P}_{1}, \mathrm{P}_{2}$ 


\section{Taxa}

Scinaia furcellata (Turner) J. Agardh

\section{Order Peyssonneliales}

\section{Family Peyssonneliaceae}

Peyssonnelia bornetii Boudouresque \& Denizot

Peyssonnelia coriacea Feldmann

Peyssonnelia dubyi P. Crouan \& H. Crouan

Peyssonnelia squamaria (S.G. Gmelin) Decaisne

\section{Order Plocamiales}

\section{Family Plocamiaceae}

Plocamium cartilagineum (Linnaeus) P.S. Dixon

Plocamium raphelisianum P.J.L. Dangeard

\section{Order Rhodymeniales}

Family Champiaceae

Champia compressa Harvey

Champia parvula (C. Agardh) Harvey

Gastroclonium clavatum (Roth) Ardissone

Gastroclonium reflexum (Chauvin) Kützing ${ }^{1}$

Family Lomentariaceae

Lomentaria articulata (Hudson) Lyngbye ${ }^{9}$

*Lomentaria articulata var. linearis Zanardini

\section{Family Rhodymeniaceae}

Botryocladia botryoides (Wulfen) Feldmann

Rhodymenia caespitosa P.J.L. Dangeard

Rhodymenia pseudopalmata (J.V. Lamouroux) P.C. Silva ${ }^{9}$

\section{Classe Stylonematophyceae}

\section{Order Stylonematales}

\section{Family Stylonemataceae}

Chroodactylon ornatum (C. Agardh) Basson

Stylonema alsidii (Zanardini) K.M. Drew

Stylonema cornu-cervi Reinsch

\section{Location in this study}

\section{Previous reference}

$\mathrm{P}_{1}$

$$
\begin{aligned}
& P_{1} \\
& P_{2} \\
& P_{1} \\
& P_{1}, P_{2}
\end{aligned}
$$

$$
P_{1}, P_{2}
$$

$1,3,4,6,7$

$\begin{array}{ll}1,2,4,7 & \\ 1,2,5,7 & P_{1} \\ 1,7 & P_{1}, P_{2}\end{array}$

$1,6,7$

7

6

$P_{1}$ 7

6,7
$\mathrm{P}_{1}$

$1,4,6,7$
$\mathrm{P}_{1}, \mathrm{P}_{2}$

$\mathrm{P}_{1}$

\section{Notes}

${ }^{1}$ The twelve species identified in this study were reported newly for the Mediterranean coast of Morocco. However, all of these species have been signaled in the Atlantic coast of Morocco by Benhissoune et al. (2001, 2002a, b, 2003). ${ }^{2}$ Ceramium giacconei was described by Cormaci and

Furnari (1991) to accommodate the entity previously known in the Mediterranean as Ceramium cingulatum Weber-van Bosse. Therefore, the single record of the later species by González-García and CondePoyales (1994), should be treated as C. giacconei. ${ }^{3}$ Reported as Ceramium nodulosum by González-García and Conde-Poyales (1994). Ceramium virgatum was cited by C. Agardh (1811) as a synonym of $C$. 
rubrum and later noted as possibly conspecific with C. nodulosum (Maggs \& Hommersand, 1993). But Silva et al. (1996) point out that $C$. nodulosum Ducluzeau, the name proposed by Maggs and Hommersand (1993) to accommodate the species commonly reported as $C$. rubrum (Hudson) C. Agardh, cannot be used. Later, Maggs et al. (2002) suggested that the name $C$. virgatum Roth should be used for $C$. nodulosum (Lightfoot) Ducluzeau.

${ }^{4}$ Barros-Barreto et al. (2006) reported that Centroceras clavulatum (C. Agardh) Montagne may consist of a species complex. Recently, Won et al. (2009) indicated that $C$. clavulatum has a biogeographic distribution limited to the Pacific Ocean. More recently, Hassoun et al. (2018) pointed out that all specimens of Centroceras examined from Morocco are not $C$. clavulatum but $C$. gasparrini. As a result, $C$. clavulatum was excluded from Moroccan flora and replaced with $C$. gasparrinii.

${ }^{5}$ Polysiphonia stricta and Ectocarpus crouanii were previously reported only in one reference in an unpublished thesis (Kazzaz, 1989). Thus, the presence of the two species in Morocco was confirmed in this study. Furthermore, this is the first record of Ectocarpus crouanii in Africa.

${ }^{6}$ Confirmation of presence in Morocco for Erythrotrichia investiens, which was reported just in the study of González-García and Conde-Poyales (1991). This is one of the rare records of this species in Africa after the first report was made by John et al. (1979).

${ }^{7}$ This is the first record of Porphyrostromium ciliare in Morocco and Africa after the only record of this species was made by Dangeard (1949) from the Atlantic.

${ }^{8}$ The two green algae and four red seaweeds present in our checklist were reported from Morocco for the second time after the investigation conducted by Riadi et al. (2011).

${ }^{9}$ Confirmation of presence in the Mediterranean coast of Morocco for ten species previously signaled in an unpublished national thesis (Kazzaz, 1989).

In this study, the results have indicated that Rhodophyta represent the dominant group with four classes (Bangiophyceae, Compsopogonophyceae, Florideophyceae and Stylonematophyceae) and 19 orders (see table 1). They encompass 207 taxa within 39 families. The family Rhodomelaceae contained the most species (38) grouped in 17 genera, followed by the Ceramiaceae with 25 species. Moreover, Ceramium (12), Gelidium (12) and Polysiphonia (11) were the most diverse genera among the red algae.

A total of 51 taxa belonging to the Ochrophyta (Phaeophyceae) were signaled in this catalogue, under 13 different families. Sargassaceae was the most diverse family among brown algae with 14 taxa, followed by the Dictyotaceae with 8 species. Additionally, the genus Cystoseira has the most taxa (12 species) among the brown algae of PNAH.

Regarding the Ulvophyceae, 48 taxa from 12 families have been reported from the coastline of the National Park of Al-Hoceima. The most species-rich family among the Ulvophyceae, was the Cladophoraceae with 17 taxa, followed by the Ulvaceae with 10 species. Furthermore, Cladophora with 11 and Ulva with 10 were the most species-rich genera in green algae of the Park.

Using present-day taxonomy, 306 taxa of marine algae have been registered from the Al-Hoceima National Park in this investigation. The data were amended and complemented by the bibliographic analysis of the following works (González-García \& Conde-Poyales, 1994 and González-García et al., 2017). Among the 306 taxa, 95 species were not found in our samples and 93 were newly reported to the Park, and the taxonomic identity of 26 taxa was amended (Figure 2).

In comparison with the checklist of seaweed of Morocco (Benhissoune et al., 2001, 2002a, 2002b, 2003), our checklist found to contain more than twothird (306 vs. 403) of the species recorded in the Mediterranean coast of Morocco, which represent a high percentage (76\%) (Figure 3).

\section{Conclusion}

In conclusion, to the best of our knowledge, this is the first inclusive seaweeds checklist of AlHoceima National Park. The seaweeds catalogue encompasses 306 taxa, which represent more than two-third of the total species reported in the Mediterranean shore of Morocco. This fact evidences that Al-Hoceima National Park

\begin{tabular}{|l|c|c|c|c|c|}
\hline & Class & Order & Family & Genus & Species \\
\hline Chlorophyta & 1 & 3 & 12 & 17 & 48 \\
\hline Ochrophyta & 1 & 6 & 13 & 23 & 51 \\
\hline Rhodophyta & 4 & 19 & 39 & 103 & 207 \\
\hline Total & 6 & 28 & 64 & 143 & 306 \\
\hline
\end{tabular}

Table 1. Number of seaweeds taxa according to different taxonomic groups (Class, order, family, genus and species). 


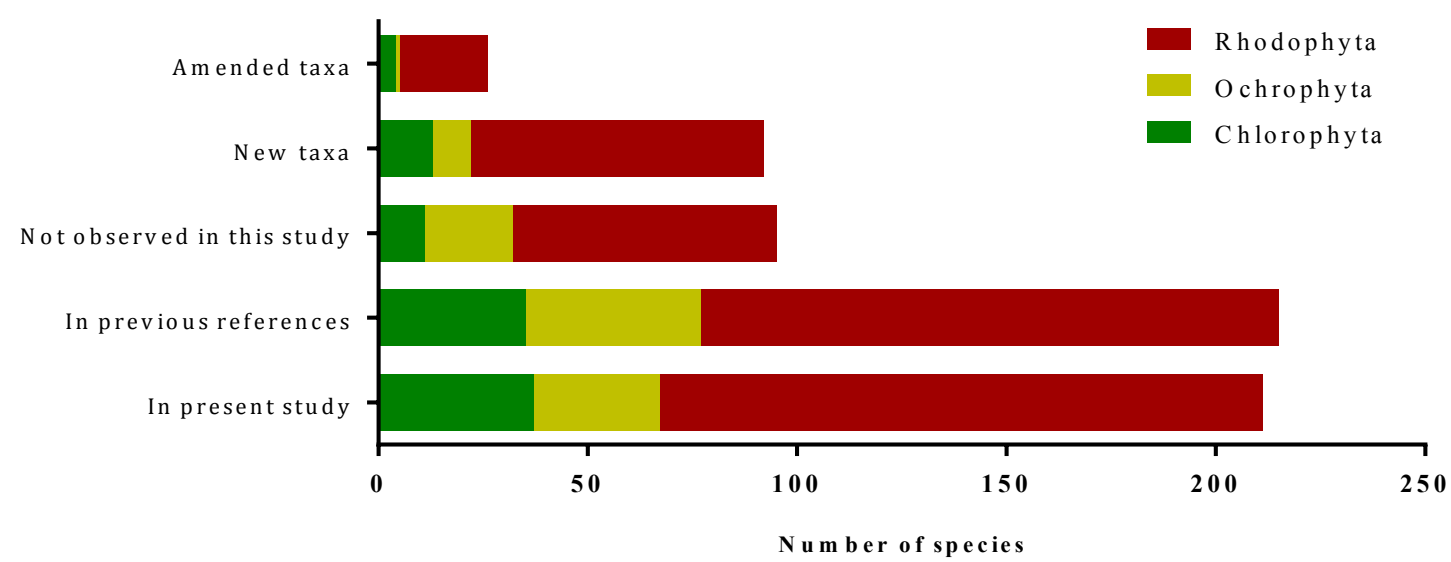

Figure 2. Detailed comparison of the PNAH seaweed flora: our study and previous works

Figura 2. Comparación detallada de la flora de algas marinas PNAH: nuestro estudio y trabajos anteriores

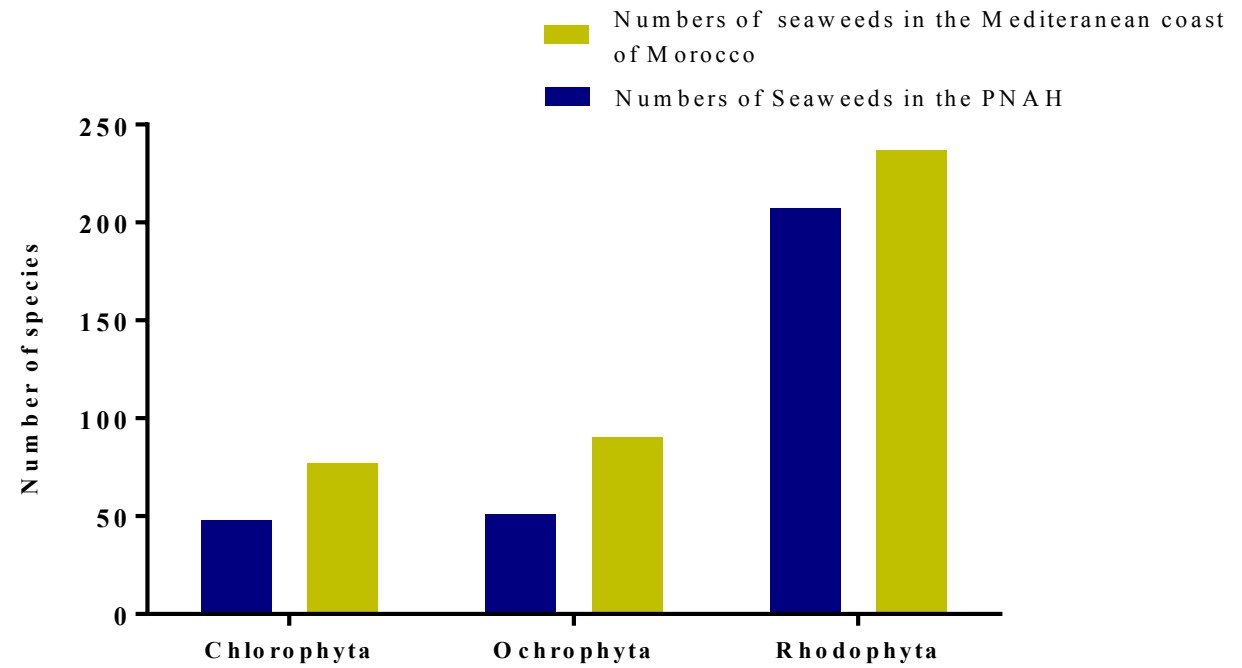

Figure 3. Numbers of species of seaweeds listed in the Mediterranean coast of Morocco (Benhissoune et al,. 2001, 2002a, b, 2003) and Al-Hoceima National Park (this study).

Figura 3. Números de especies de algas marinas identificadas en la costa Mediterránea de Marruecos (Benhissoune et al,. 2001, 2002a, b, 2003) y en el Parque Nacional de Al-Hoceima (En nuestra investigación).

of Morocco has a great diversity of species. Moreover, this study showcases the need for more phycological studies in other localities along the Mediterranean coast of Morocco, in order to clarify their algal biodiversity.

Publishing this checklist will improve its accessibility to the international society. Furthermore, the seaweed diversity information provided in this catalogue could serve as an infrastructure for future phycological investigations of the taxa in this Specially Protected Area of Mediterranean Importance.

\section{Acknowledgements}

The authors are indebted to the RODPALorganization, especially Mr. Hakim Messaoudi, for their support with the administrative and logistic assistance during the expeditions to Al-Hoceima National Park. We thank Mr. Hamid Kaouass for his collaboration in the production of the PNAH map. We express our gratitude towards Dr Michael J. Wynne (University of Michigan) for identified, gave complementary information and for supplying the hard-to-find literature about the newly species. Special thanks are given to Dr. Michael Guiry for his help with retrieving the taxonomic status of some species. 


\section{References}

Agardh, C. A. 1811 [1810-1812]. Dispositio algarum Sueciae... Pp 17-26. Litteris Berlingianis Lundae [Lund].

Altamirano, M., de la Rosa, J., Zanolla, M., Souza-Egipsy, V. \& Diaz, J. (2010). New records for the Benthic Marine Flora of Chafarinas Islands (Alborán Sea, Western Mediterranean). Acta Botanica Malacitana, 35, 165-167.

Ballesteros, E. (1990). Check-list of benthic marine algae from Catalonia (north-west Mediterranean). Treballs de l'Institut Botànic de Barcelona, 13, 5-52.

Barros-Barreto, D., Beatriz, M., Mclvor, L., Maggs, C. A. \& Gomes Ferreira, P. C. (2006). Molecular systematics of Ceramium and Centroceras (Ceramiaceae, Rhodophyta) from Brazil. Journal of Phycology, 42, 905-921.

Benhissoune, S., Boudouresque C. F. \& Verlaque, M. (2001). A check-list of marine seaweeds of the Mediterranean and Atlantic coasts of Morocco. I. Chlorophyceae. Botanica Marina, 44, 171-182.

Benhissoune, S., Boudouresque C. F. \& Verlaque, M. (2002a). A check-list of marine seaweeds of the Mediterranean and Atlantic coasts of Morocco. II. Phaeophyceae. Botanica Marina, 45, 217-230.

Benhissoune, S., Boudouresque, C. F., PerretBoudouresque, M. \& Verlaque M. (2002b). A checklist of the seaweeds of the Mediterranean and Atlantic coasts of Morocco. III. Rhodophyceae (excluding Ceramiales). Botanica Marina, 45, 391-412.

Benhissoune, S., Boudouresque, C. F., PerretBoudouresque, M. \& Verlaque M. (2002b). A checklist of the seaweeds of the Mediterranean and Atlantic coasts of Morocco. IV. Rhodophyceae Ceramiales. Botanica Marina, 46, 55-68.

Bueno del Campo, I. \& González-García, J. A. (1996). Guía marina de la región de Melilla. Colección Ensayos Melillenses. Fundación Municipal Sociocultural. Excmo. Ayuntamiento de Melilla, Melilla, 276 pp.

Coll, M., Piroddi, C., Steenbeek, J., Kaschner, K., Ben Rais Lasram, F., Aguzzi, J., Ballesteros, E., Bianchi, C. N., Corbera, J., Dailianis, T., Estrada, M., Froglia, C., Galil, B. S., Gasol, J.M., Gertwagen, R., Gil, J., Guilhaumon, F., Kesner-Reyes, K., Kitsos, M. S., Koukouras, A., Lampadariou, N., Laxamana, E., López-Fé de la Cuadra, C. M., Lotze, H. K., Martin, D., Mouillot, D., Oro. D, Raicevich, S., Rius-Barile, J., SaizSalinas, J. I., San Vicente. C., Somot, S., Templado, J., Turon, X., Vafidis, D., Villanueva, R. \& Voultsiadou, E. (2010). The Biodiversity of the Mediterranean Sea: Estimates, Patterns, and Threats. PLOS ONE, 5 (8), e11842. doi:10.1371/journal.pone.0011842

Conde Poyales, F. (1984). Contribución al conocimiento de la flora algal bentónica del Mar de Alborán, Islas Chafarinas. Acta Botanica Malacitana, 9, 41-46.

Conde Poyales, F. (1989). Ficogeografía del mar de Alborán en el contexto del Mediterráneo occidental. Anales del Jardín Botánico de Madrid, 46, 21-26.

Cormaci, M. \& Furnari, G. (1991). The distinction of Ceramium giacconei sp. nov. (Ceramiales, Rhodophyta) in the Mediterranean Sea from Ceramium cingulatum. Cryptogamie, Algologie, 12 (1), 43-53.

Cormaci, M., Furnari, G. \& Alongi, G. (2014). Flora marina bentonica del Mediterraneo: Chlorophyta. Bollettino delle sedute della Accademia Gioenia di Scienze Naturali in Catania, 47, 11-436.

Cormaci, M., Furnari, G., \& Alongi, G. (2017). Flora marina bentonica del Mediterraneo: Rhodophyta (Rhodymeniophycidae escluse). Bollettino Accademia Gioenia di Scienze Naturali in Catania, 50 (380), 1-391.

Cormaci, M., Furnari, G., Giaccone, G. \& Serio, D. (2004). Alien macrophytes in the Mediterranean Sea: a review. Recent Research Developments in Environmental Biology, 1, 153- 202.

Cormaci, M., Furnari, G., Catra, M., Alongi, G. \& Giaccone, G. (2012). Flora marina bentonica del Mediterraneo: Phaeophyceae. Bollettino dell' Accademia Gioenia di Catania, 45 (375), 1-508.

Dangeard, P. (1949). Les algues marines de la côte occidentale du Maroc. Le Botaniste, 34, 89-189.

Flores-Moya, C. \& Conde Poyales, F. (1998). Nuevas citas de macroalgas marinas para las islas Chafarinas. Acta Botanica Malacitana, 23, 197-199.

Furnari, G., Giaccone, G., Cormaci, M., Alongi, G. \& Serio, D. (2003). Marine biodiversity of Italian coasts: catalogue of the macrophytobenthos. Biologia Marina Mediterranea, 10 (1), 3-482.

Gómez Garreta, A., Gallardo, T., Ribera, M.A., Cormaci, M., Furnari, G., Giaccone, G. \& Boudouresque, C. F. (2001a). Check-list of Mediterranean Seaweed. III. Rhodophyceae Rabenh. 1. Ceramiales Oltm. Botanica Marina, 44 (5), 425-460

González-García, J.A. (1994). La flora marina de Melilla. Colección Ensayos Melillenses. Fundación Municipal Sociocultural. Excmo. Ayuntamiento de Melilla, Melilla, $212 \mathrm{pp}$.

González García, J. A. \& Conde Poyales F. (1994). Catálogo del macrofitobentos del Mediterráneo de Marruecos. Acta Botanica Malacitana, 19, 5-27.

González García, J. A. \& Conde Poyales, F. (1991). Estudio florístico, fenológico, autoecológico y fitogeográfico del macrofitobentos de la Mar Chica (Sebcha Buareg de Nador, Mediterráneo Marroquí). Acta Botanica Malacitana, 16, 63-80.

González García, J. A., Paredes Ruiz, P. \& Mirón, C. E. (2017). Catálogo de Macroalgas de los Peñones de Alhucemas y vélez de la gomera (Mediterráneo Occidental, Mar de Alborán). Algas. Boletín Informativo de la Sociedad Española de Ficología, 53, 33-38.

Guiry, M.D. \& Guiry, G.M. (2018). AlgaeBase. World-wide electronic publication. National University of Ireland, Galway. http://www.algaebase.org. (Assessed 27 November 2018).

Hassoun, M., Wynne, M.J., Moussa, H., Salhi, G., Zbakh, H., Riadi, H. \& Kazzak, M. (2018). An investigation of members of the tribe Ceramieae (Ceramiaceae, Rhodophyta) occurring on the Mediterranean and Atlantic shores of Morocco. Algae, 33(3), 243-267. https://doi.org/10.4490/algae.2018.33.8.29

Hassoun, M., Salhi, G., Bouksir, H., Moussa, H., Riadi, H. \& Kazzak, M. (2014). Codium tomentosum var. mucronatum et son epiphyte Aglaothamnion pseudobyssoides, deux nouvelles espèces d'algues benthiques pour la phycoflore du Maroc. Acta Botanica 
Malacitana, 39, 37-44.

Hassoun, M., Moussa, H., Salhi, G., Zbakh, H., Riadi, H. \& Kazzaz, M. (2018). New records of benthic marine algae from Morocco. Acta Botanica Malacitana, 43. http://dx.doi.org/ 10.24310/abm.v43i0.3265

Hassoun, M., Moussa, H., Zbakh, H., Riadi, H., Kazzaz, M. \& Salhi, G. (2015). First record of Polyneura bonnemaisonii (Delesseriaceae, Rhodophyta) from the Atlantic Ocean of Africa. Marine Biodiversity Records, 8, e139. https://doi.org/10.1017/S1755267215001190

Hassoun, M., Salhi, G., Kazzaz, M., Riadi, H. \& Moussa, H. (2016a). New records of Polysiphonia sensu lato from the Atlantic Ocean in Morocco. Marine Biodiversity, 46(2), 443-449. https://doi.org/10.1007/ s12526-015-0387-8

Hassoun, H., Salhi, G., Moussa, H., Riadi, H., Kazzaz, M. \& Zbakh, H. (2016b). Ceramium cornutum and Ceramium pallidum (Rhodophyta: Ceramiales) two new records for Morocco: morphology and reproductive structures. Botany Letters, 163 (1), 25-31.

John, D.M., Price, J.H., Maggs, C.A. \& Lawson, G.W. (1979). Seaweeds of the western coast of tropical Africa and adjacent islands: a critical assessment. III. Rhodophyta (Bangiophyceae). Bulletin of the British Museum (Natural History) Botany, 7, 69-82.

Katsanevakis, S., Acar, Ü., Ammar, I., Balci, B.A., Bekas, P., Belmonte, M., Chintiroglou, C. C., Consoli, P., Dimiza, M., Fryganiotis, K., Gerovasileiou, V., Gnisci, V., Gülşahin, N., Hoffman, R., Issaris, Y., IzquierdoGomez, D., Izquierdo-Munoz, A., Kavadas, S., Koehler, L., Konstantinidis, E., Mazza, G., Nowell, G., Önal, U., Özen, M. R., Pafilis, P., Pastore, M., Perdikaris, C., Poursanidis, D., Prato, E., Russo, F., Sicuro, B., Tarkan, A. N., Thessalou-Legaki, M., Tiralongo, F., Triantaphyllou, M., Tsiamis, K., Tunçer, S., Turan, C., Türker, A. \& Yapici, S. (2014). New Mediterranean biodiversity records. Mediterranean Marine Science, 15(3), 675-695.

Kazzaz, M. (1989). Contribution à l'étude de la flore algale marine de la région Ouest de la Méditerranée. MSc Thesis. Université Mohammed V, Morocco, 246 pp.

Maggs, C.A., Ward, B.A., Mclvor, L.M., Evans, C.M., Rueness, J. \& Stanhope, M.J. (2002). Molecular analyses elucidate the taxonomy of fully corticated, nonspiny species of Ceramium (Ceramiaceae, Rhodophyta) in the British Isles. Phycologia, 41, 409420.

Maggs, C.A. \& Hommersand, M.H. (1993). Seaweeds of the British Isles Vol. 1. Rhodophyta Part A, 3. Ceramiales. Natural History Museum, London, 444 pp.

Moussa, H., Wynne, M. J., Hassoun, M., Salhi, G., Zbakh, H. Kazzaz, M. \& Riadi, H. (2015). On the occurrence of three red algal species new to the Mediterranean Sea in Al-Hoceima National Park (Morocco). Botanica Marina, 58 (6): doi: http://dx.doi.org/10.1515/bot2015-0048.

Navarro, M. J. \& Gallardo. T. (1989). Aportación al conocimiento de la flora bentónica de las costas mediterráneas africanas occidentales. Botanica complutensis, 15, 203-214.

Ni-Ni-Win, Hanyuda, T., Draisma, S.G.A, Furnari, G., Meinesz, A. \& Kawai, H. (2011). Padina ditristromatica sp. nov. and Padina pavonicoides sp. nov. (Dictyotales, Phaeophyceae), two new species from the Mediterranean Sea based on morphological and molecular markers. European Journal of Phycology, 46 (4), 327-341.

PNUE-PAM-CAR/ASP, (2009). Report of Meeting of MED Pol Focal Points. UNEP (DEPI)/MED WG. 337/ inf.6. Meeting of MAP Focal Points, Athens, Greece, $97 \mathrm{pp}$.

Riadi, H., Salhi, G., Boukssir, H., Moussa, H. \& Hassoun, M. (2013). Acrosymphyton purpuriferum (J. Agardh) Sjöstedt et Balliella cladoderma (Zanardini) Athanasiadis, deux nouvelles espèces d'algues benthiques de la Méditerranée marocaine. Bulletin de I'Institut Scientifique, Rabat, Section Sciences de la Vie, 35, 9-14.

Riadi, H., Salhi, G., Saoud, Y., Kadiri, M. \& Kazzaz. M. (2011). Nuevas aportaciones a la flora algal de las costas marroquíes. Acta Botanica Malacitana, 36, 161-163.

Ribera, M. A., Garreta, A.G., Gallardo, T., Cormaci, M., Furnari, G. \& Giaccone, G. (1992). Check-list of Mediterranean seaweeds. I. Fucophyceae (Warming, 1884). Botanica marina, 35 (2), 109-130.

Salhi, G., Hassoun, M., Moussa, H., Zbakh, H. \& Riadi, H. (2016). First record of Rhodymenia holmesii (Rhodymeniaceae, Rhodophyta) for the Mediterranean Sea from Morocco. Marine Biodiversity Records, 9:62.

Salhi, G., Hassoun, M., Moussa, H., Zbakh, H., Kazzaz, M., \& Riadi, H. First record of the red alga Tiffaniella gorgonea: an introduced species in the Mediterranean Sea. Botanica Marina. Doi: https://doi.org/10.1515/ bot-2017-0129

Tsiamis, K., Panayotidis, P., Economou-Amilli, A. \& Katsaros, C. (2013). Seaweeds of the Greek coasts. I. Phaeophyceae. Mediterranean Marine Science, 14, 141-157.

Tunesi, L., Mo, G., Salvati, E., Molinari, A., Rais, C. Limam, A., Benhamza, A., Benhissoune, S., Nachite, D., Zeichen, M., Agnesi, S., Di Nora, T., Piccione, M. E., Bazairi, H. \& Sadki, I. (2003). Rapport global des travaux de prospection de la partie marine du Parc National d'Al-Hoceima. Projet Régional pour le Développement d'Aires Protégées Marineset Côtières dans la Région Méditerranéenne (Projet MedMPA). CAR/ASPPAM-PNUE, $111 \mathrm{pp}$.

Limam, A., Benhamza, A., Benhissoune, S., Nachite, D., Zeichen, M., Agnesi, S., Di Nora, T., Piccione, M. E., Bazairi, H. \& Sadki, I. (2003). Rapport global des travaux de prospection de la partie marine du Parc National d'Al-Hoceima. Projet Régional pour le Développement d'Aires Protégées Marineset Côtières dans la Région Méditerranéenne (Projet MedMPA). CAR/ASPPAM-PNUE. $111 \mathrm{pp}$.

Verlaque, M., Ruitton, S., Mineur, F. \& Boubouresque, C.F. (2015). CIESM Atlas of Exotic Species in the Mediterranean. Vol. 4. Macrophytes. CIESM Publishers, Monaco, $362 \mathrm{pp}$.

Won, B.Y., Cho, T.O. \& Fredericq, S. (2009). Morphological and molecular characterization of species of the genus Centroceras (Ceramiaceae, Ceramiales), including two new species. Journal of Phycology, 45, 227-250. 